\title{
A Coupling Control Model of Color Temperature and Illumination in Naturally Lighted Room Based on Evolutionary Algorithm
}

\author{
Qingcheng Lin, Caizi Luo, Huiling Cai, and Hui Xiao* \\ College of Electronic and Information Engineering, Tongji University, \\ 4800 Cao'an Highway, Shanghai 201804, P. R. China
}

(Received April 24, 2019; accepted May 29, 2019)

Keywords: indoor light environment comfort, coupling control method, multiobject optimization algorithm

Indoor light environment comfort is evaluated using the illumination index, and the color temperature of building space is not specifically a requirement. However, previous studies have demonstrated that color temperature has a great impact on the human central nervous system, and regulating it can not only improve brain function, but also promote brain fatigue recovery. Owing to natural light dynamic change characteristics, the illumination and color temperature of building space that introduce natural light present a complex nonlinear coupling relationship. Moreover, the regional and seasonal characteristics of architecture aggravate the difficulty in the dynamic management and control of an architectural light environment. Thus, intelligent control technologies for natural lighting and artificial lighting are gradually attracting the attention of researchers in the field of intelligent building. However, coupling control methods for illumination and color temperature are not mature, with many engineering issues unresolved. Therefore, a new coupling model based on coupling parameters and synchronous control is proposed in this paper. The distribution uniformity between illumination and color temperature can be met simultaneously in a dynamic light environment. This work has an active role in the improvement of indoor light environment quality and further supplements the light quality evaluation index.

\section{Introduction}

Intelligent buildings can be expected to provide a highly efficient, convenient, comfortable, and energy-saving perfect indoor space environment. ${ }^{(1,2)}$ On the basis of the complete interconnection of electrical and electronic equipment, intelligent buildings can predefine a program in response to information from various sensors and efficiently manage light, temperature, ventilation, and so forth, so as to meet the needs of its owners. ${ }^{(3)}$ One of the goals of constructing intelligent buildings is to use resources more efficiently and reduce a building's negative impact on the environment. ${ }^{(4-6)}$ Presently, light energy consumption represents a significant percentage, which contributes up to $20 \%$ of the world's total energy usage. ${ }^{(7)}$ In

*Corresponding author: e-mail: xiaohui@tongji.edu.cn https://doi.org/10.18494/SAM.2019.2414 
office buildings, in particular, the energy consumed by artificial lighting can be up to $40 \%$ of the total energy consumption. ${ }^{(8)}$

Daylighting can be considered a very important strategy in replacing artificial lighting. ${ }^{(9-11)}$ A sensor incorporated smart LED illuminating system has been used for environments that require different lighting levels due to a daylight factor. ${ }^{(12)}$ The existing research results showed that the introduction of daylight-linked controls can reduce light energy consumption up to $60 \%$, with values ranging from 20 to $40 \% .{ }^{(13-16)}$ Simulation showed that the introduction of natural light into the sample space lighting environment can reduce the power consumption of artificial lighting by 50 to $80 \%$. ${ }^{(17)}$

Additionally, the existing research studies have indicated that the light quality affects the moods of its users and therefore their comfort and productivity; thus, visual comfort plays a significant role in determining the quality of life of occupants in buildings. ${ }^{(18-20)}$ It has been theoretically verified that different color temperature light sources have different effects on the inhibition of melatonin secretion, as well as on sleep status, behavior, and subjective wakefulness. ${ }^{(21-23)}$ Brief exposure to strong natural light indoor may reduce afternoon sleepiness, ${ }^{(24)}$ whereas exposure to more sunlight during workdays increases physical activity and sleep duration. ${ }^{(25)}$ A long-term deprivation of natural light makes people's circadian rhythms more unstable, ${ }^{(26)}$ whereas too much sunlight may cause glare. Thus, it is essential to maintain a comfortable working environment; accordingly, shading devices, such as Venetian blinds, are required. ${ }^{(27,28)}$ The lighting coefficient function, attenuation function, and illumination characteristic value have been used to select the louver angle and modulate the artificial light proportion. ${ }^{(29)}$

As indicated by the above findings, energy saving performance can be improved by combining natural light with artificial light in a single control system while maintaining visual comfort. However, by comparing previous research studies that mainly focus on illumination, ${ }^{(17,29)}$ it was found that, when light sources of various color temperatures, particularly natural light, are introduced, the imparity of the color temperature of a light source and human visual becomes subsistent. In the study of indoor light environment control combined with natural light, the control of the light environment is mostly limited to the illumination control of the indoor illuminated surface, without the study of environmental color temperature. ${ }^{(12)}$ In this paper, an indoor light environment control strategy that combines hybrid illuminance with hybrid color temperature is proposed, considering daylight provision, visual comfort, and light energy use.

\section{Hybrid Color Temperature}

A natural daylight factor is important in natural daylighting. For a particular building, the natural daylight factor is associated with the sun shading measures and depth. When the sun shading measure of the office space is a louver in a certain depth indoor, the natural daylight factor is a function of the louver angle.

There is a matrix of natural daylight factor functions in this paper. Each element is a natural daylight factor function. Thus, the attenuation ratio is calculated at a depth of $0.1 \mathrm{~m}$ as the origin. ${ }^{(29)}$ 


$$
d\left(x_{i}\right)=\frac{1.756}{x+1.756}
$$

The total illuminance of $m$ lamps at a certain calculation point is determined by the superposition of the illuminance of each lamp at the point.

$$
E_{m}=\sum_{j=1}^{m} K_{j} \Phi_{j},
$$

where $E_{m}$ is the total illuminance at the point when there are $m$ lamps indoor. $K_{j}$ is the proportional coefficient matrix of $j$ th lamps. Moreover, $\Phi_{j}$ is the luminous flux of $j$ th lamps.

The total hybrid illuminance $E$ at the point includes daylight illuminance and artificial illuminance, the calculation method of which is shown as

$$
E=E_{d}+E_{m} .
$$

On the basis of 1931 CIE chromaticity coordinates and Glassman's law, when $n$ light sources have different colors, ${ }^{(30)}$

$$
X_{m}=\sum_{k=1}^{n} X_{k}, Y_{m}=\sum_{k=1}^{n} Y_{k}, Z_{m}=\sum_{k=1}^{n} Z_{k},
$$

where $X_{m}, Y_{m}$, and $Z_{m}$ are the tristimulus values of the hybrid light sources. $X_{k}, Y_{k}$, and $Z_{k}$ are the tristimulus values of the $k$ th light source. The relationship between the tristimulus value and the chromaticity coordinates is shown as

$$
\left\{\begin{array}{l}
x=\frac{X}{X+Y+Z}, \\
y=\frac{Y}{X+Y+Z},
\end{array}\right.
$$

where $(x, y)$ are the chromaticity coordinates. According to Eqs. (4) and (5), the chromaticity coordinates of hybrid light can be calculated as

$$
\left\{\begin{array}{c}
x_{m}=\frac{X_{m}}{X_{m}+Y_{m}+Z_{m}}=\frac{\sum_{j=1}^{n} \frac{x_{k}}{y_{k}} Y_{k}}{\sum_{j=1}^{n} \frac{1}{y_{k}} Y_{k}}, \\
y_{m}=\frac{Y_{m}}{X_{m}+Y_{m}+Z_{m}}=\frac{\sum_{j=1}^{n} Y_{k}}{\sum_{j=1}^{n} \frac{1}{y_{k}} Y_{k}},
\end{array}\right.
$$


where $\left(x_{m}, y_{m}\right)$ are the chromaticity coordinates of hybrid light and $\left(x_{k}, y_{k}\right)$ are the chromaticity coordinates of the $k$ th hybrid light. $Y$ is both the tristimulus value and brightness of the light source. The relationship between the brightness $L$ of the ideal diffuse reflector and the illuminance of the diffuse reflector is shown as

$$
L=\frac{E}{\pi}
$$

Thus, Eq. (6) can be

$$
\left\{\begin{array}{c}
x_{m}=\frac{\sum_{j=1}^{n} \frac{x_{k}}{y_{k}} E_{k}}{\sum_{k=1}^{n} \frac{1}{y_{k}} E_{k}} \\
y_{m}=\frac{\sum_{j=1}^{n} E_{k}}{\sum_{k=1}^{n} \frac{1}{y_{k}} E_{k}} .
\end{array}\right.
$$

The equation used for calculating the hybrid color temperature is ${ }^{(31)}$

$$
T_{m}=449 A^{3}+3525 A^{2}+6823.3 A+5520.33,
$$

where $T_{m}$ is the color temperature of hybrid light sources and $A$ is the reciprocal of the isothermal slope.

$$
A=\frac{x_{m}-0.3320}{y_{m}-0.1858}
$$

The chromaticity coordinates of the light sources $A$ and $B$ are $\left(x_{A}, y_{A}\right)$ and $\left(x_{B}, y_{B}\right)$ respectively. When $E_{A}=K E_{B}$, the illuminances $E_{A}$ and $E_{B}$ of the light sources $A$ and $B$ respectively, can be calculated as

$$
\left\{\begin{array}{l}
K=\frac{x_{m}-x_{B}}{x_{A}-x_{m}} \cdot \frac{y_{A}}{y_{B}}, \\
E_{A}=\frac{K}{1+K} \cdot E_{m}, \\
E_{B}=E_{m}-E_{A} .
\end{array}\right.
$$




\section{Strategies of Lighting Control}

In the sample space, the illuminance at a calculation point is provided by natural light (color temperature is $T_{\text {out }}$ ) and 2700 and $6700 \mathrm{~K}$ lamps. There are 15 lamps of 2700 and 6700 K. Every two lamps form a group. The lamps are symmetrical about the median line of the window; thus, symmetrical lamps obtain the same output. To sum up, the objective function can be expressed by the mathematical model as

$$
\begin{gathered}
\min \left\{\begin{array}{l}
f_{1}=\left(\operatorname{avg}(E)-E_{g}\right)^{2}, \\
f_{2}=(U(E)-1)^{2}, \\
f_{3}=\left(\operatorname{avg}\left(T_{m}\right)-T_{g}\right)^{2}, \\
f_{4}=\left(U\left(T_{m}\right)-1\right)^{2},
\end{array}\right. \\
0 \% \leq \phi_{L i}, \phi_{H i} \leq 100 \%, i=1,2,3, \ldots, 10 .
\end{gathered}
$$

Here, $E_{i j}$ is the hybrid illumination matrix.

$$
E_{i j}=E_{d}+E_{L}+E_{H}
$$

Here, $E_{d}$ is the daylight illumination matrix, $E_{L}$ is the artificial illumination matrix of lamps with a color temperature of $2700 \mathrm{~K}$, and $E_{H}$ is the artificial illumination matrix of lamps with a color temperature of $6700 \mathrm{~K}$. $T_{i j}$ is the hybrid apparent color temperature matrix whose calculation method is shown in Eq. (9). To obtain convergence rapidly, all objective functions are evaluated using the square variance.

In conclusion, this problem is a multiobjective optimization problem in finding multiple solutions in a feasible domain. Evolutionary computation is a mature global optimization method with high robustness and wide applicability with the characteristics of self-organization, self-adaptation, and self-learning. It can effectively deal with complex problems that are difficult to be solved by traditional optimization algorithms without being limited by the nature of the problem. In addition, evolutionary algorithms are often used in the optimization of multiobjective problems, which are generally called evolutionary multiobjective optimization algorithms (MOEAs). Therefore, the differential evolution (DE) algorithm, one of the evolutionary algorithms, is adopted in this paper to solve the value satisfying the objective function. ${ }^{(32)}$ When $E_{\text {out }}$ is less than $E$ th, there is no natural light in the room. Eth is set as $51 \mathrm{x}$. The color temperature of all lamps is set as the given average color temperature $T_{g}$.

The outdoor color temperature is $T_{\text {out }}$, the average color temperature is $T_{g}$, the color temperature of the indoor calculation point is $T_{i j}$, and the uniformity of color temperature is $U_{c t}=$ the minimum color temperature/average color temperature. There are $\forall i, T_{\text {out }}$ $<T_{i}<T_{g}$ or $T_{g}<T_{i}<T_{\text {out }}$ and also $\min \left(T_{i}\right)<\operatorname{avg}\left(T_{i}\right)<\max \left(T_{i}\right)$; when $T_{g}<T_{\text {out }}$, there is $T_{g}<\min \left(T_{i}\right)<\operatorname{avg}\left(T_{i}\right)<\max \left(T_{i}\right)<T_{\text {out }}$, so 


$$
\frac{T_{g}}{T_{\text {out }}}<\frac{T_{g}}{\operatorname{avg}\left(T_{i}\right)}<\frac{\min \left(T_{i}\right)}{\operatorname{avg}\left(T_{i}\right)}=U_{T}
$$

Similarly, when $T_{\text {out }}<T_{g}$, there is

$$
\frac{T_{\text {out }}}{T_{g}}<\frac{T_{\text {out }}}{\operatorname{avg}\left(T_{i}\right)}<\frac{\min \left(T_{i}\right)}{\operatorname{avg}\left(T_{i}\right)}=U_{T} .
$$

From Eqs. (13) and (14),

$$
\frac{\min \left(T_{\text {out }}, T_{g}\right)}{\max \left(T_{\text {out }}, T_{g}\right)}<\frac{\min \left(T_{i}\right)}{\operatorname{avg}\left(T_{i}\right)}=U_{T} .
$$

The distance from the window is inversely proportional to daylight illumination. The daylight illumination at the point closest to the window is approximately equal to the given average illumination $E_{g}$. The light coefficient at the point closest to the window is $E_{g} / E_{\text {out }}$.

The optimization results are evaluated using the following four indexes: average illumination, uniformity of illumination, average color temperature, and uniformity of color temperature. In this paper, the evaluation criterions are as follows: the average illuminance of all calculation points in the calculated grid is within the given illuminance $E_{g} \pm 10 \%$, the uniformity of illuminance is greater than 0.6 , the average color temperature is within the given color temperature $T_{g} \pm 10 \%$, and the uniformity of color temperature is greater than 0.9 .

\section{Results}

In this paper, the office space is simplified into an independent space model $\left(9 \times 5 \times 3 \mathrm{~m}^{3}\right)$. The window area is $5 \times 3 \mathrm{~m}^{2}$, with the window facing south and being single-side-lighted. The installation height of the lamps is $3.5 \mathrm{~m}$, as shown in Fig. 1. Two lamps with color temperatures

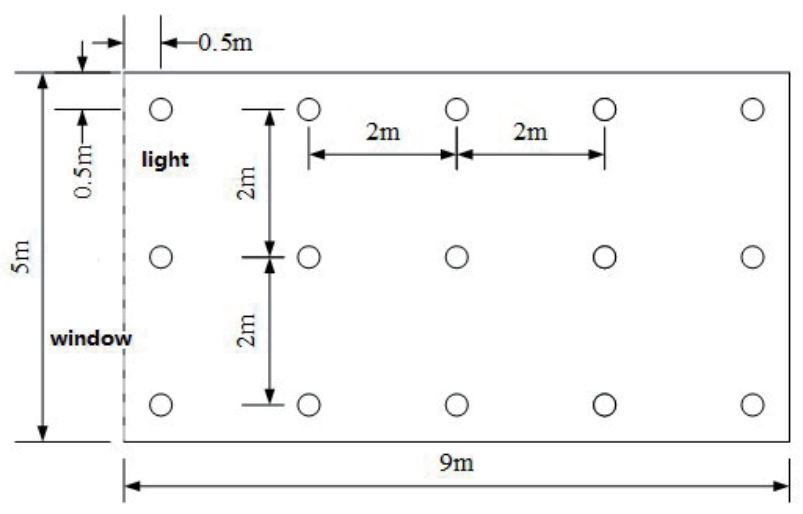

Fig. 1. Lamp layout location diagram. 
of 2700 and $6700 \mathrm{~K}$ are installed at each position. The lamp flux is adjustable, so as to obtain a wider range of color temperatures. Starting from the point $0.5 \mathrm{~m}$ away from the window, there is a point every $0.5 \mathrm{~m}$ increase in distance from the window and every $0.5 \mathrm{~m}$ in the direction parallel to the window, and the grid height of illumination calculation points is $0.75 \mathrm{~m}$.

Under the condition that the outdoor illuminance (3000 1x), target color temperature $(5000 \mathrm{~K})$, and outdoor color temperature $(6000 \mathrm{~K})$ remain constant, the target illuminance is $450 \mathrm{~lx}$. The results of the optimization of the multiobjective DE algorithm for four objective functions are shown in Fig. 2. The population number is 70 and the number of iterations is 100 . The global optimum is found around generation 30 .

The results of the optimization of the multiobjective DE algorithm in four objective functions, obtained after the restriction of the experimental vector entering the population was relaxed, are shown in Fig. 3. It can be seen that the performance of the optimal individuals in the population in each generation differs in the four objective functions. According to the relaxed rules, comparing pairs of individuals, if the value of the individual for no less than 1 objective function is better than that of the compared individual, the former may replace the compared individual. In choosing the optimal individual history vector and optimal vector population history, this comparison method increases the population diversity and broadens the population search scope, but without the optimal individual history, it is incapable of keeping the best individual of the last generation. Owing to a poorer convergence effect, stable performance is not achieved in a multiobjective function. The iterative results of the two selection strategies for the objective functions are shown in Table 1.

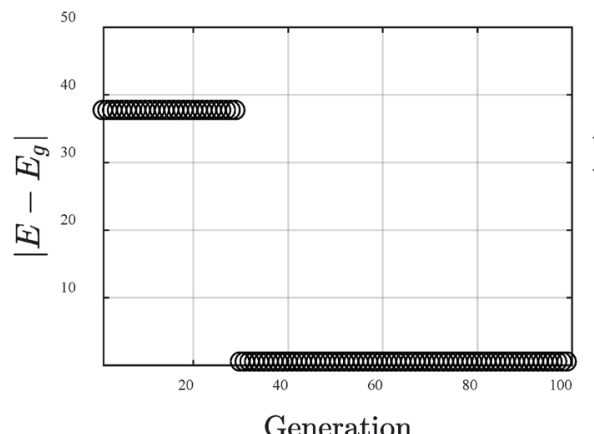

(a)

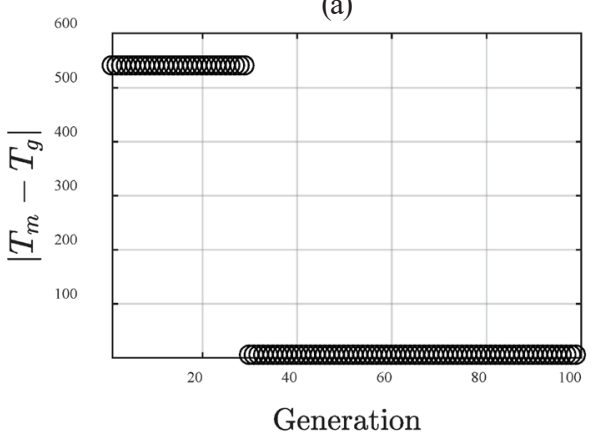

(c)

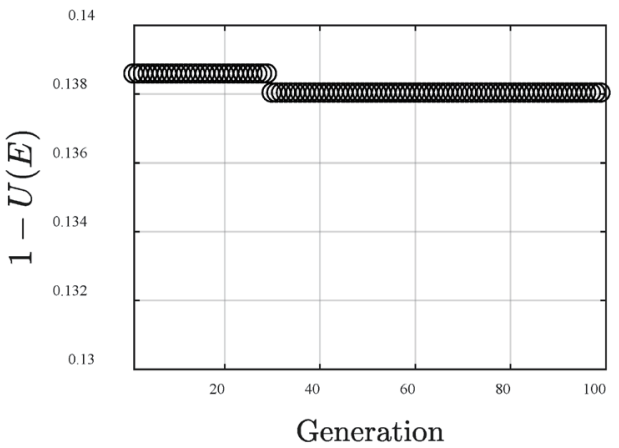

(b)

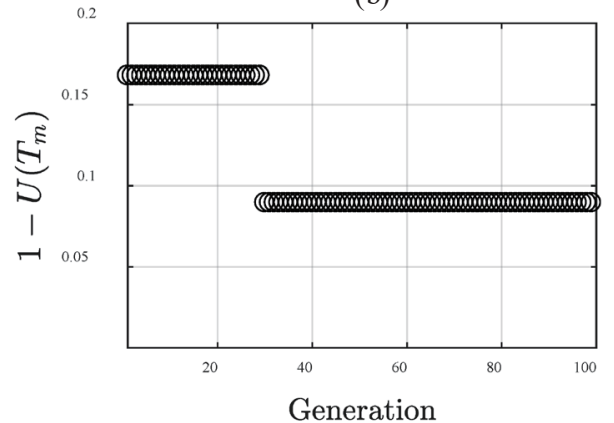

(d)

Fig. 2. Results of objective function optimization. 


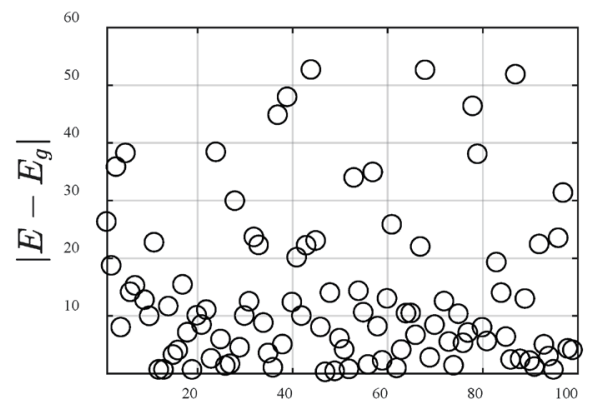

Generation

(a)

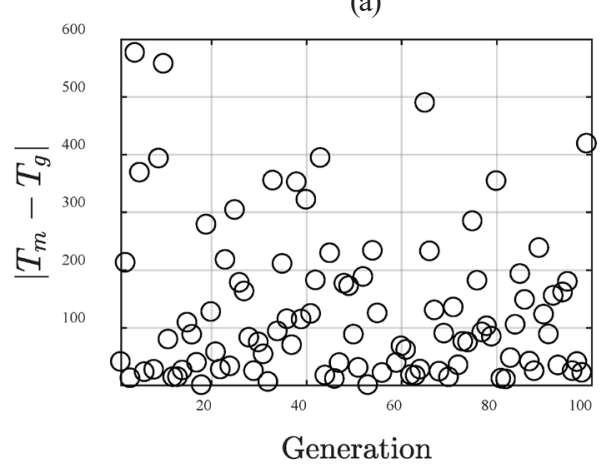

(c)

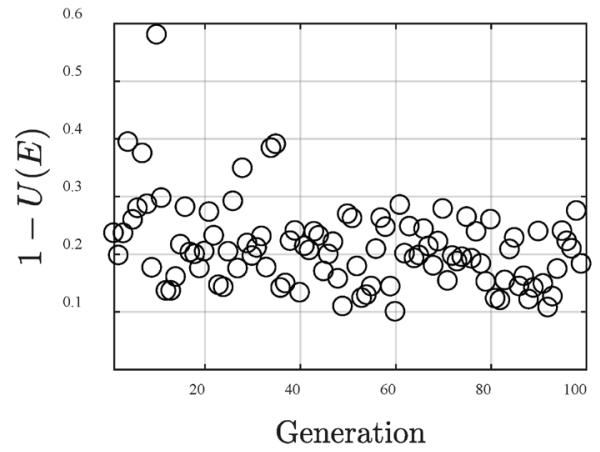

(b)

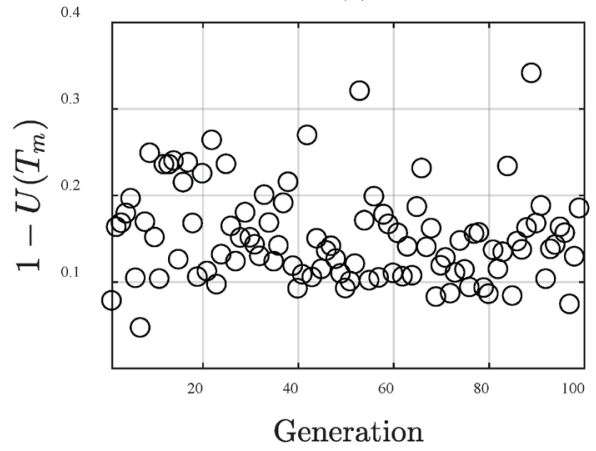

(d)

Fig. 3. Results of objective function optimization of not completely inferior selection strategy.

Table 1

Optimization results with different strategies.

\begin{tabular}{lcc}
\hline Strategy & Rigid not inferior to & Incompletion inferior to \\
\hline Average illuminance (lx) & 450.0 & 466.66 \\
Uniformity of illuminance & $\mathbf{0 . 8 6}$ & 0.77 \\
Average color temperature (K) & 4995 & 4769 \\
Uniformity of color temperature & $\mathbf{0 . 9 1}$ & 0.90 \\
\hline
\end{tabular}

Accordingly, the former selection strategy is chosen to calculate the optimization results of the multiobjective DE algorithm when the outdoor illuminance (3000 1x), target color temperature $(5000 \mathrm{~K})$, and outdoor color temperature $(6000 \mathrm{~K})$ remain constant, and the target illuminance is 450 lx, as shown in Fig. 4. The results of calculating the objective functions with different target illuminances are shown in Table 2. The difference between the target illuminance and the calculated average illuminance is maximum at $12.2 \mathrm{~lx}$ when the target illuminance is $500 \mathrm{~lx}$. It is proved that the multiobjective DE algorithm can optimize the standard luminous flux and color temperature, and make the average illuminance reach the target illuminance, while other indexes also meet the requirements. 


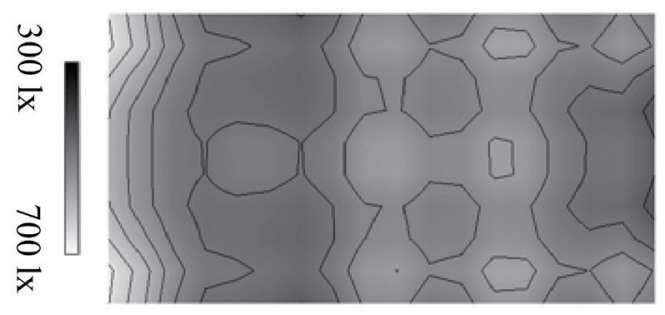

(a) Hybrid illuminance with $E_{\text {desire }} 500$ lx.

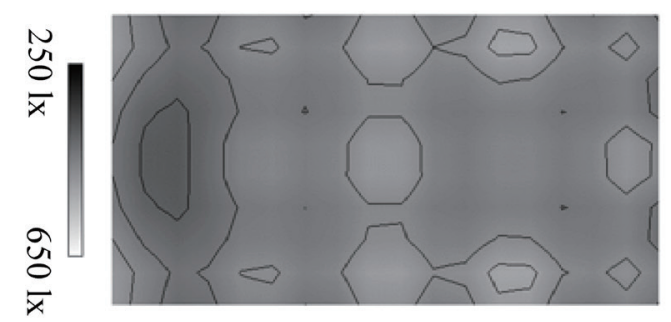

(c) Hybrid illuminance with $E_{\text {desire }} 450$ lx.

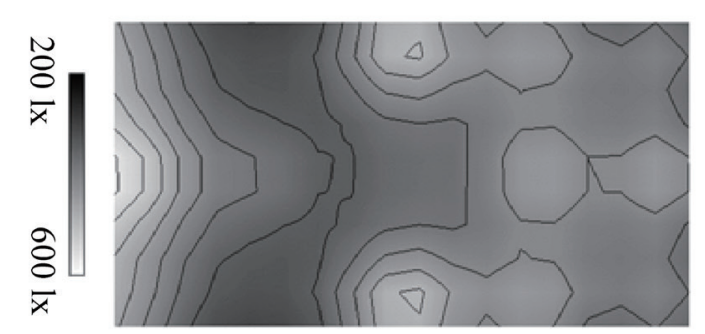

(e) Hybrid illuminance with $E_{\text {desire }} 400$ lx.

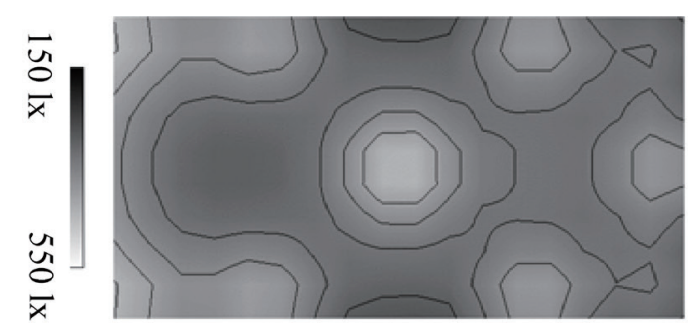

(g) Hybrid illuminance with $E_{\text {desire }} 350$ lx.

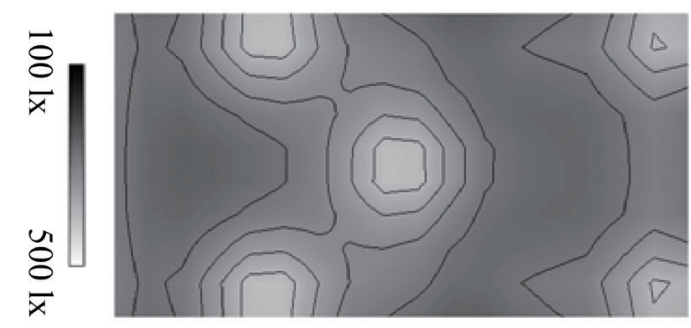

(i) Hybrid illuminance with $E_{\text {desire }} 300$ lx.

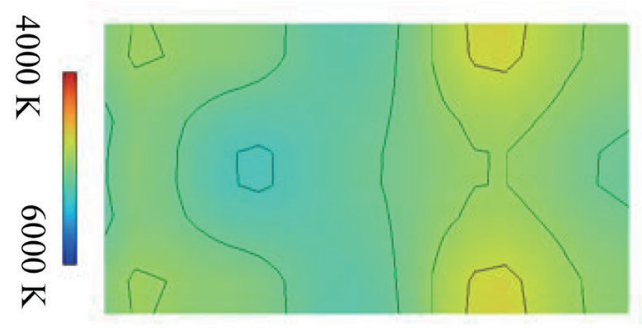

(b) Hybrid color temperature with $E_{\text {desire }} 500$ 1x.

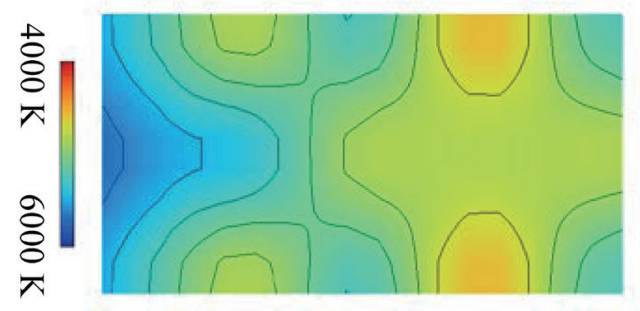

(d) Hybrid color temperature with $E_{\text {desire }} 450$ lx.

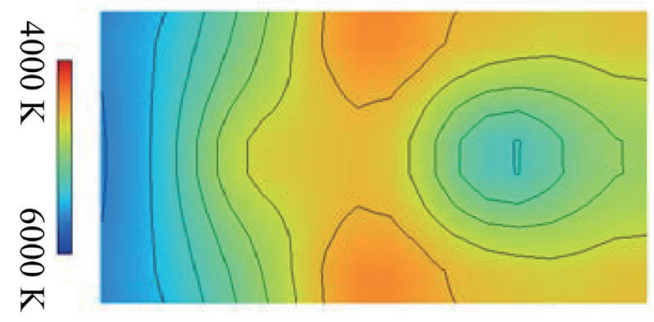

(f) Hybrid color temperature with $E_{\text {desire }} 4001 \mathrm{x}$.

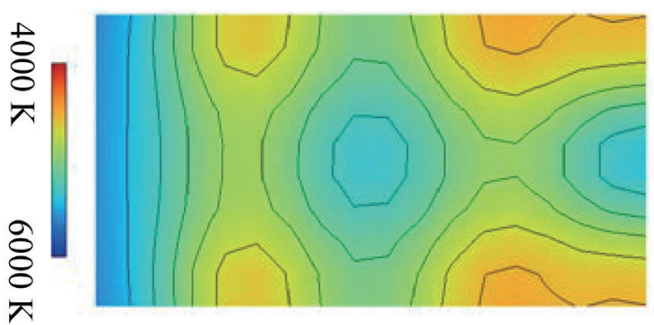

(h) Hybrid color temperature with $E_{\text {desire }} 350$ lx.

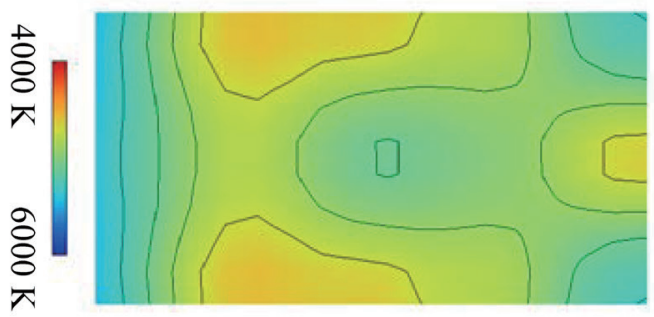

(j) Hybrid color temperature with $E_{\text {desire }} 300$ lx.

Fig. 4. (Color online) Hybrid illumination and hybrid color temperature. 
Table 2

Results of calculating objective functions with different target illuminances.

\begin{tabular}{lccccc}
\hline \multirow{2}{*}{ Evaluation parameter } & \multicolumn{5}{c}{ Target illuminance (lx) } \\
\cline { 2 - 6 } & 500 & 450 & 400 & 350 & 300 \\
\hline Average illuminance (lx) & 512.2 & 450.0 & 399.6 & 345.3 & 304.3 \\
Uniformity of illuminance & 0.86 & 0.86 & 0.77 & 0.80 & 0.81 \\
Average color temperature (K) & 5010 & 4995 & 4890 & 4978 & 4927 \\
Uniformity of color temperature & 0.93 & 0.91 & 0.90 & 0.90 & 0.93 \\
\hline
\end{tabular}

\section{Conclusions}

Dynamic daylight intensity and color temperature affect the hybrid illuminance indoor light environment and hybrid color temperature on the working surface. To describe the phenomenon that the difference between the daylight color temperature and the color temperature of lamps leads to different visual colors on an indoor working surface better, this paper puts forward the concept of hybrid color temperature and its calculation method. The hybrid illumination and hybrid color temperature at calculation points on an indoor working surface can be calculated using daylight and artificial illumination models. In this paper, the lighting control strategy is proposed, and the control modes under different conditions are given. The control strategy is applied to an independent spatial model $\left(9 \times 5 \times 3 \mathrm{~m}^{3}\right)$. The results show that the control strategy makes the indoor light environment meet the requirements of average illumination and the uniformity of illumination, and at the same time, the average color temperature and the uniformity of hybrid color temperature also exhibit good performance.

In this paper, the problem of uneven illumination and color temperature is considered in an indoor light environment after the introduction of daylight and artificial light, and the corresponding control method is proposed. However, there is no relevant research on the minimum requirement of the uniformity of color temperature in the light environment and the extent to which the uniformity of color temperature affects the visual comfort and psychological response of users in the light environment. In this paper, the control method of environmental variables is given. Combined with the subsequent research, this control method can be improved further.

\section{Acknowledgments}

This work is partially supported by the Fundamental Research Funds for the Central Universities (22120180189 and 22120180299).

\section{References}

1 Z. Chen, F. Wang, and Q. Feng: Energy Build 128 (2016) 484.

2 A. Ghaffarianhoseini, H. AlWaer, and A. Ghaffarianhoseini: Intell. Build. Inter. 10 (2018) 122.

3 A. Kumar and A. Singh: IEEE Sens. J. 18 (2018) 4847.

4 I. Sartori, A. Napolitano, and K. Voss: Energy Build. 48 (2012) 220. 
T. A. Nguyen and M. Aiello: Energy Build. 56 (2013) 244.

6 M. Sechilariu, B. Wang, and F. Locment: Energy Build. 59 (2013) 236.

7 C. Atici, T. Ozcelebi, and J. Lukkien: IEEE Trans. 57 (2011) 788.

8 L. Pérez-Lombard, J. Ortiz, and C. Pout: Energy Build. 40 (2008) 394.

9 A. Eltaweel and S. Yuehong: Energy Build. 152 (2017) 137.

10 H. Poirazis, A. Blomsterberg, and M. Wall: Energy Build. 40 (2008) 1161.

11 R. Delvaeye, W. Ryckaert, and L. Stroobant: Energy Build. 127 (2016) 969.

12 M. Ali Özc: Inf. Syst. 13 (2017) 61.

13 T. de Rubeis, M. Muttillo, L. Pantoli, and I. Nardi: Energy Build. 152 (2017) 24.

14 C. Yin, X. Huang, and S. Dadras: Inf. Sci. 465 (2018) 38.

15 I. Ullah, H. Lv, A. J.-W. Whang, and Y. Su: Energy Build. 154 (2017) 19.

16 L. Sedki and M. Maaroufi: Energy Build. 152 (2017) 434.

17 M. Bodart and A. D. Herde: Energy Build. 34 (2002) 421.

18 M. A. Fasi and I. M. Budaiwi: Energy Build. 108 (2015) 307.

19 X. Wang and J.-P. M. G. Linnartz: Light. Res. Technol. 49 (2017) 603.

20 A. Steidle, L. Werth, J. de Boer, and K. Sedlbauer: Proc. Experiencing Light (2014) 78.

21 I. M. Iskra-Golec, A. Wazna, and L. Smith: Light. Res. Technol. 44 (2012) 506.

22 J. L. Souman, A. M. Tinga, and S. F. Te Pas: Behav. Brain Res. 337 (2018) 228.

23 A. Sivaji, S. Shopian, and Z. M. Nor: Procedia - Social Behav. Sci. 97 (2013) 638.

24 K. Kaida, M. Takahashi, and T. Haratani: Sleep 29 (2006) 462.

25 M. Boubekri, I. N. Cheung, and K. J. Reid: J. Clin. Sleep Med. 10 (2014) 603.

26 A. Kawasaki, S. Wisniewski, and B. Healey: Sci. Rep. 8 (2018) 1.

27 S. Zhang and D. Birru: Sol. Energy 86 (2012) 860.

28 Y. C. Chan and A. Tzempelikos: Sol. Energy 98 (2013) 241.

29 X. Chen, H. Xiao, and J. Fan: Chinese Control and Decision Conference (CCDC, 2015) 805.

30 D. Malacara: The International Society for Optical Engineering: Bellingham (2002).

31 C. S. Mccamy: Color Res. Appl. 17 (1992) 142.

32 A. Mandal, A. Das, and P. Mukhejee: IEEE Trans. Evol. Comput. (CEC, 2011).

\section{About the Authors}
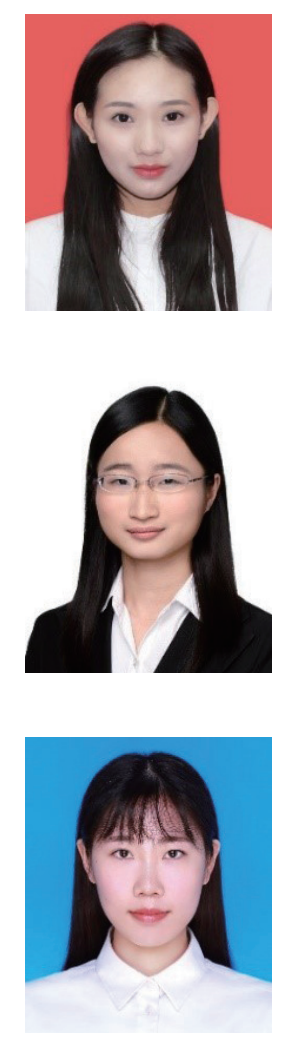

Qingcheng Lin received her B.S. degree in mechanical design and manufacturing and automation from Northeastern University, Shenyang, China, in 2018. She is currently pursuing her Ph.D. degree in control theory and control engineering in the Department of Control Science and Engineering, Tongji University, Shanghai, China. Her research interests include machine learning and intelligent control.

Caizi Luo received her B.S. degree in automation from Tongji University, Shanghai, China, in 2016. Since 2016, she has been studying at Tongji University for her M.S degree. Her research interests include electrical lighting and intelligent control.

Huiling Cai received her B.S. degree in automation from Jiangnan University, Wuxi, China, in 2017. She is currently pursuing her Ph.D. degree in control theory and control engineering in the Department of Control Science and Engineering, Tongji University, Shanghai, China. Her research interests include electrical lighting and intelligent control. 


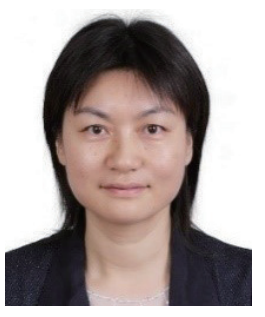

Hui Xiao received her B.S., M.S., and Ph.D. degrees in control theory and control engineering from the Department of Control Science and Engineering, Tongji University, Shanghai, China, in 1992, 1998, and 2007, respectively. From 1992 to 1997, she was a lecturer at Tongji University. From 1997 to 2011, she was an associate professor at Tongji University. Since 2011, she has been a professor at Tongji University. Her research interests include intelligent building, intelligent control, life optics, and novel application techniques. 